\title{
Study of Cerebrovascular Disease at Dhulikhel Hospital
}

\author{
Pokharel B.R*, Pant $\mathrm{P}^{*}$, Gurung $\mathrm{R}^{*}$, Koju $\mathrm{R}^{*}$, Bedi T. R.S*, Pathak $\mathrm{M}^{*}$
}

\section{ABSTRACT}

A stroke is the rapidly developing loss of brain function due to disturbance in the blood supply to the brain. It is a medical emergency that requires rapid diagnosis and treatment. The availability of CT Scan has made it easier for early diagnosis and intervention. The aim of this study was to identify the risk factors (eg. hypertension, diabetes mellitus and cigarette smoking) commonly seen in patients admitted in the hospital with stroke along with age group, sex distribution, duration of stay in the hospital and the occupancy of stroke patients admitted in medical ward. A total of 51 stroke patient were admitted in medical ward in the review period. CT scan was used to differentiate hemorrhagic from ischemic stroke. Patients were analyzed in terms of risk factors (like hypertension, diabetes mellitus and cigarette smoking) along with age and sex. In case of ischemic stroke along with CT Scan, ECG, Echocardiography, Carotid Doppler was done accordingly. Out of 2307 total admissions in medical ward, 51 cases $(2.2 \%)$ were stroke patients. Among the stroke cases, $59 \%$ constituted male and $41 \%$ female. Similarly around $25 \%$ of the total cases were Diabetic / Hypertensive both or alone, $35 \%$ of the total cases were smokers, $48.6 \%$ of the cases were in the $51-70$ age groups. $61 \%$ stayed in the hospital for 5 days or less. Cerebrovascular disease carries a lot of financial, physical, social and emotional implications. If we can address the modifiable risk factors, we can reduce the incidence of stroke.

Correspondence:

Dr Babu Ram Pokharel Lecturer

Email : drbrpokh@gmail.com

Kathmandu University hospital 


\section{INTRODUCT ION}

Risk factors for stroke include advanced age, hypertension (high blood pressure), previous stroke or transient ischemic attack (TIA), diabetes, high cholesterol, cigarette smoking and atrial fibrillation. ${ }^{1}$ High blood pressure is the most important modifiable risk factor of stroke. Cerebro Vascular Accident (CVA) is a burden to medical practitioners as well as for the patient. If risk factors are evaluated and managed CVA can be avoided. CVA is becoming one of the leading causes of death. Stroke will soon be the most common cause of death worldwide. Stroke is the third leading cause of death in the western word after heart disease and cancer ${ }^{2}$, and causes $10 \%$ of worldwide deaths. ${ }^{3}$ The hallmark of stroke is abrupt onset of neurologic deficit that corresponds to interruption of vascular supply to specific brain tissue.

Atherosclerotic vascular disease usually affects the large vessels. Diabetes mellitus and hypertension are known risk factor for CVA. The LDL particle present in diabetic persons is more atherogenic than non diabetic patient. It has been said that diabetes is equivalent to prior $\mathrm{Ml}$ in terms of vascular disease.

Prior TIA history is important in case of thrombotic stroke. Embolic source can from either cardiac or carotid in origin. ${ }^{4}$ Common symptom in stroke is weakness, paralysis (one side of the body with partial or complete loss of voluntary movement or sensation in a leg or arm), there is possibility of speech problems and weak face muscles, causing drooling of saliva, and numbness or tingling.

A stroke can affect balance, vision, swallowing, breathing and even cause unconsciousness. In cases of severe brain damage there may be deep coma, paralysis of one side of the body, loss of speech, followed by death. Permanent neurological deficit may occur even after recovery.

Small infarcts are associated with poorly controlled hypertension or diabetes and have been found in several clinical syndromes, including contralateral pure motor or pure sensory deficit, ipsilateral ataxia with crural paresis, and dysarthria with clumsiness of the hand. ${ }^{5}$

\section{MATERIALS AND METHODS}

A hospital based study was carried out in medical ward in the review period (2008 June to 2009 June). The total number of patient admitted in medical ward was 2307, out of which 51 were stroke patient. These 51 patients were analyzed in the regard of risk factors (like hypertension, diabetes mellitus and cigarette smoking) along with age, sex and days of hospital stay. Almost all the patient attended had been subjected to CT Scan of head, ECG, Echocardiography and Carotid Doppler accordingly on need basis.

\section{RESULTS}

On the basis of the study of cerebrovascular disease done at Dhulikhel Hospital, CVA constituted about $2 \%$ of total number of patient admitted in medical ward in the review period (51 out of 2307).

Reviewing the CVA in terms of sex distribution, it was found that male were more prone for stroke than female. Male accounted for $59 \%$ of total stroke patient admitted where as female accounted about $41 \%$ which almost correlates with the series from other countries as well.

Smoking is one of the risk factor for CVA. Most of the smokers have a tendency of denying their smoking habit. Only $29.5 \%$ of the total CVA patient admitted that they are smoking currently, while $5.8 \%$ admitted that they smoked in the past. The study showed that around $35.2 \%$ of total cases have declared smoking whether in the past or the present habit.

Table 1: Frequency of smokers in stroke patients

\begin{tabular}{lcc} 
Smoking & Frequency & Percent \\
\hline Yes & 15 & 29.5 \\
No & 3 & 5.8 \\
Left & 3 & 5.8 \\
Non responders & 30 & 58.9 \\
Total & 51 & $100 \%$
\end{tabular}

The next most common risk factor for CVA is hypertension. On analyzing the history of CVA 
patient admitted around $22 \%$ of the patient were hypertensive while $4 \%$ of the patient had both hypertension and diabetes.

Table 2: Past medical history in stroke patients

$\begin{array}{lcc}\text { Past History } & \text { Frequency } & \text { Percent } \\ \text { Non responders } & 16 & 31.4 \\ \text { HTN } & 11 & 21.6 \\ \text { HTN/DM } & 2 & 3.9 \\ \text { No } & 20 & 39.2 \\ \text { Seizure disorder } & 2 & 3.9 \\ \text { Total } & 51 & 100\end{array}$

The study showed the most vulnerable age for developing stroke is around $51-70$ years of age, in the review period around $49 \%$ of the total CVA patients were of this age distribution, followed by 71 years above and in between 31 to 50 years of age which constitute of around $19 \%$ of total patients each. The average hospital stay for the stroke patients is around 5 days which is $61 \%$ followed by $6-10$ days (26\%).

Table 3: Duration of hospital stay

\begin{tabular}{ccc}
\hline Hospital Stay & Frequency & Percent \\
$\leq 5$ & 21 & 61 \\
6 to 10 & 9 & 25.4 \\
11 to 15 & 2 & 5.8 \\
\hline 16 to 20 & 1 & 3.9 \\
Missing & 2 & 3.9 \\
\hline Total & 51 & 100 \\
\hline
\end{tabular}

\section{DISCUSSION}

Stroke quite often regarded as struck by the hand of God. ${ }^{1}$ In many cases stroke is preventable provided that the risk factors are treated timely. Once CVA develops it is a burden for the patients and the relatives. The residual neurologic damage after CVA makes the situation more difficult.
As seen in the study about $2.2 \%$ of hospital admission belongs to CVA with an average stay of about 5 to 10 days. Most of the cases requires intense imaging and further tests which creates financial burden to the patients and their family members. During the course of treatment and after the discharge patients are physically handicapped and not able to carry out their day to day works. In fact many of the patients do have emotional problems like denial and depression. Many of the patients do not admit that they smoke. It requires a repeated questionings to find out their smoking and drinking habit. This is one of the habits which should be strongly discouraged. Men smoke more than women.

However in Nepal women are exposed to indoor pollution mainly from household smoke exposure during cooking. Due to changes in social and economic condition of the people, changes in life style, changes in eating habits also contributed to increase incidence of hypertension and diabetes. The study showed around one fourth of stroke patients had hypertension or diabetes in combination or alone. Good control of hypertension and diabetes definitely decreases the stoke incidence. Stroke can occur at any age according to risk factors. The study showed fifth and sixth decade of life had high incidence CVA. It may be due increase incidence of hypertension in this age group.

\section{CONCLUSION}

Stroke cases are commonly seen in many people these days, it makes their life miserable and hard to manage day to day work for the patient and their family members. It needs a lot of financial, family, social and emotional support for the patient. There are factors which helps people to minimize the incidences like by avoiding smoking and drinking habit, proper control of blood pressure and diabetes to live a good and healthy life. 


\section{REFERENCES}

1. Adams HP et al: Guidelines for the early management of patients with ischemic stroke: $A$ scientific statement from the Stroke Council of the American Stroke Association. Stroke 34:1056, 2003 [PMID: 12677087]

2. Feigin VL (2005). "Stroke epidemiology in the developing world". Lancet 365 (9478): 2160-1. doi:10.1016/S0140-6736(05)667554. PMID 15978910.

3. The World health report 2004. Annex Table 2:
Deaths by cause, sex and mortality stratum in WHO regions, estimates for 2002.. Geneva: World Health Organization

4. Kleindorfer D et al: Incidence and short-term prognosis of transient ischemic attack in a population-based study. Stroke 36:720, 2005 [PMID: 15731465]

5. Lastilla M. Lacunar infarct. Clin Exp Hypertens. 2006 Apr-May;28(3-4):205 15. [PMID: 16833026] 